\title{
Trapeziectomy with Ligament Reconstruction and Interposition Arthroplasty Using the Palmaris Longus Tendon: An Average 5-Year Follow-up
}

\author{
Daisuke Kawamura, MD, Tadanao Funakoshi, MD*, Norimasa Iwasaki, MD \\ Department of Orthopaedic Surgery, Faculty of Medicine and Graduate School of Medicine, Hokkaido University, Sapporo, \\ ${ }^{*}$ Keiyu Orthopaedic Hospital, Tatebayashi, Japan
}

Background: Trapeziectomy with ligament reconstruction and tendon interposition (LRTI) with the flexor carpi radialis (FCR) tendon is one of the most common procedures for the treatment of trapeziometacarpal osteoarthritis. We modified the LRTI, using the palmaris longus (PL) tendon instead of the FCR tendon. The aim of this retrospective study was to evaluate the clinical outcomes of trapeziectomy with our modified LRTI procedure at a mean follow-up of 5 years.

Methods: Fourteen thumbs in 13 patients (12 women) with a mean age of 64 years (range, 50 to 77 years) were available for assessment for a mean duration of 62 months (range, 41 to 97 months). The patients were evaluated subjectively and objectively.

Results: The modified LRTI procedure provided good pain relief, motion, strength, and stability without any severe complications related to the PL tendon harvesting. Radiography showed that compared to the preoperative status, the trapezial space decreased by about $40 \%$ at the final follow-up.

Conclusions: The modified LRTI procedure provided significant subjective and objective improvements without severe complications particularly related to the harvesting of the PL tendon. This procedure is a valuable surgical option for trapeziometacarpal osteoarthritis and could be a useful salvage modality if the FCR tendon ruptures during the conventional LRTI procedure.

Keywords: Thumb, Carpometacarpal joints, Osteoarthritis, Arthroplasty

There are many different surgical procedures for treating trapeziometacarpal osteoarthritis. Despite many reports regarding these modalities, the superiority of any one procedure has not been proved. Trapeziectomy with ligament reconstruction and tendon interposition (LRTI) is one of the most common procedures for treating trapeziometacarpal osteoarthritis. Although the benefit of ligament reconstruction is still controversial, some hand surgeons, including us, believe it necessary to perform ligament reconstruction in order to obtain long-term stability at the

Received January 9, 2019; Accepted May 6, 2019

Correspondence to: Daisuke Kawamura, MD

Department of Orthopaedic Surgery, Faculty of Medicine and Graduate School of Medicine, Hokkaido University, Kita 15 Nishi 7, Kita-ku, Sapporo, Hokkaido 060-8648, Japan

Tel: +81-11-706-5937, Fax: +81-11-706-6054

E-mail: dkawa@pop06.odn.ne.jp base of the thumb after trapeziectomy. ${ }^{1,2}$

Burton and Pellegrini ${ }^{3)}$ reported on the use of trapeziectomy with LRTI using a half-strip of the flexor carpi radialis (FCR) tendon. Thereafter, the use of the full thickness of the FCR tendon was reported by Tomaino et al. ${ }^{4)}$ Previous articles have reported excellent results of pain relief and functional recovery after the conventional LRTI procedure with the FCR tendon. ${ }^{4-7)}$ However, the use of a half-FCR tendon could result in complications such as the tendon rupture, pulling discomfort in the line of the FCR tendon, and tendon adhesions. ${ }^{4,8-10)}$ There is also a risk of the tendon rupture of the FCR during the preparation of a half-strip of the FCR or the trapeziectomy. We hypothesized that the use of the palmaris longus (PL) tendon instead of the FCR tendon for LRTI could provide similar clinical results and preclude these complications. This study aimed to evaluate the clinical outcomes and adverse 
Kawamura et al. Ligament Reconstruction and Tendon Interposition Using the Palmaris Longus Tendon

Clinics in Orthopedic Surgery • Vol. 11, No. 4, 2019• www.ecios.org

effects of trapeziectomy with our modified LRTI procedure over a mean follow-up of 5 years with a minimum of 42 months.

\section{METHODS}

This study was approved by our Institutional Review Board of Hokkaido University Hospital (IRB No. 0140467), and written informed consent was waived because of the retrospective design. Fourteen thumbs in 13 consecutive patients underwent the modified LRTI procedure between 2008 and 2013 for advanced trapeziometacarpal arthritis of Eaton and Glickel grade III or IV. ${ }^{11)}$ One man and 12 women, with a mean age of 64 years (range, 50 to 77 years) were available for clinical and radiological follow-up over a mean duration of 62 months (range, 41 to 97 months). The indication for surgery was severe basal thumb pain that was nonresponsive to conservative treatments with splints, nonsteroidal anti-inflammatory drugs, and steroid injections for at least 6 months.

\section{Surgical Technique}

In the current study, we modified the LRTI described by Burton and Pellegrini. ${ }^{3)}$ Our modifications were to use a PL tendon instead of a FCR tendon for ligament reconstruction and to fill the trapeziectomy space by a tendon ball with a bone core.

An approximately $4-\mathrm{cm}$ longitudinal skin incision was made at the junction of the glabrous and non-glabrous skin over the trapeziometacarpal joint. Branches of the superficial radial nerve and the deep branch of the radial artery were carefully retracted. The dorsal capsule of the trapeziometacarpal joint was cut longitudinally between the abductor pollicis longus and the extensor pollicis brevis tendon. Total trapeziectomy was carefully performed in order not to injure the FCR tendon. The FCR tendon insertion was exposed at the base of the second metacarpal bone. The PL tendon was harvested using a tendon stripper through a small transverse skin incision at the wrist crease. A bone tunnel was created using a $2.9-\mathrm{mm}$ drill through the dorsal cortex of the first metacarpal bone to the trapezium fossa. The distal end of the PL tendon was delivered through the bone tunnel of the first metacarpal bone and interweaved into the FCR at the site just proximal to its insertion (Figs. $1 \mathrm{~A}$ and $\mathrm{B}, 2 \mathrm{~A}$ and $\mathrm{B}$ ). The other end of the PL tendon was pulled tightly, sutured to the periosteum, and interweaved to itself in the trapezial void (Fig. 1C). The remaining proximal end of the PL tendon was unrolled in transverse directions to form a sheet. The resected trapezium was divided to make a bone core and was subsequently rolled up with the PL sheet. The made-up tendon ball with a bone core was placed into the trapeziectomy space (Fig. 2C) and sutured to the adjacent volar capsule with a 3-0 nonabsorbable suture (Fig. 1D). The tendon ball with a bone core was used in all patients but the first three cases in this study. The dorsal capsule
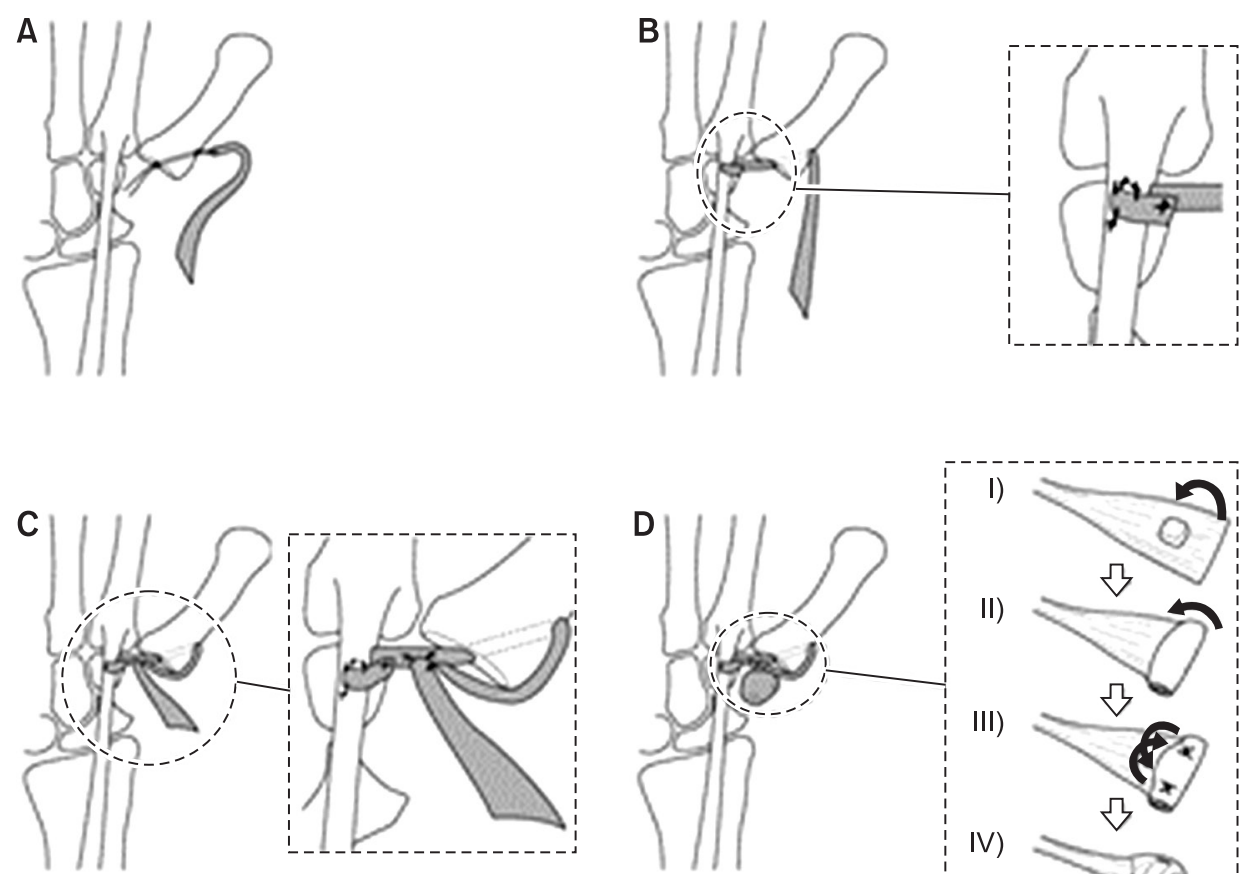

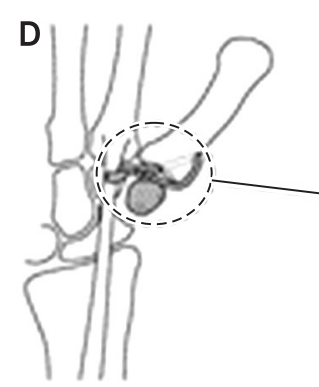

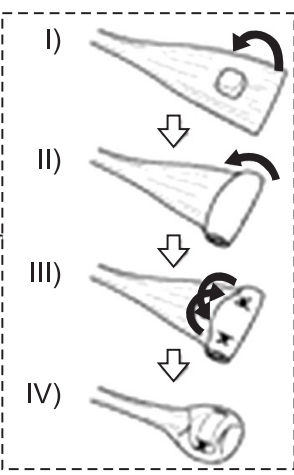

Fig. 1. Schema showing our modified ligament reconstruction and tendon interposition arthroplasty. (A) The distal end of the palmaris longus (PL) tendon was delivered through the bone tunnel at the first metacarpal bone. (B) The distal end of the PL tendon was sutured to the flexor carpi radialis with an interlacing suture at the site just proximal to its insertion. (C) The other end of the PL tendon was sutured to the periosteum and interweaved to itself in the trapeziectomy space. (D) I: The remaining proximal end of the PL tendon was unrolled in transverse direction to form a sheet. II-IV: The bone core was rolled up with the PL sheet to make a tendon ball with a bone core. The trapeziectomy space was filled with the bone-core tendon ball (curved arrows). 

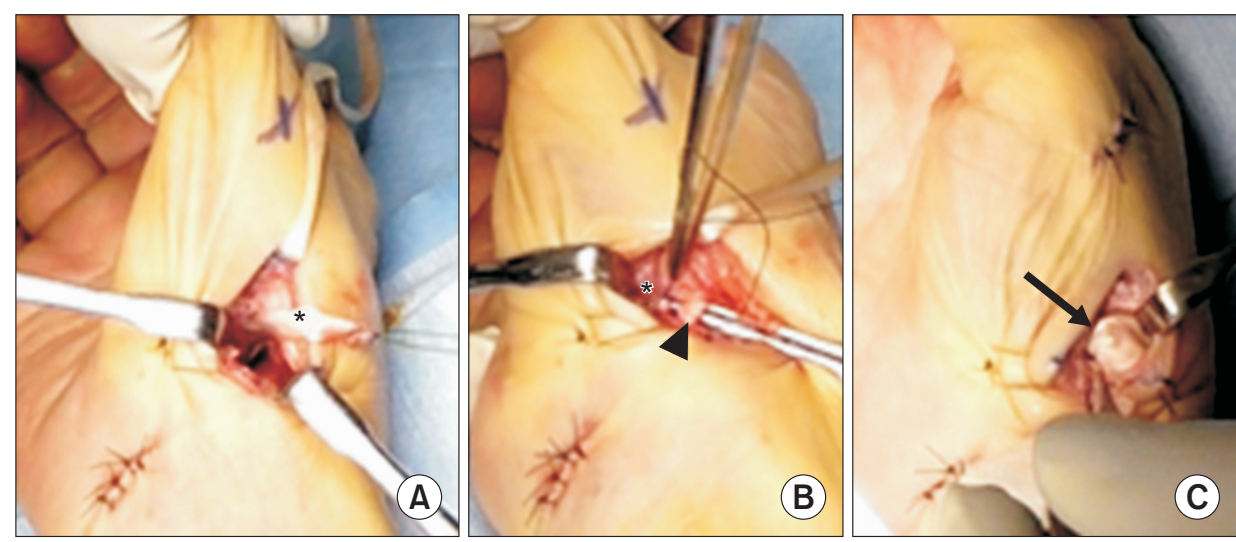

Fig. 2. Intraoperative photographs. (A) The distal end of the palmaris longus (PL) tendon (asterisk) was passed through the bone tunnel at the first metacarpal bone. (B) The distal end of the PL tendon (asterisk) was passed through the flexor carpi radialis (arrowhead) by using a tendon passer. (C) The trapeziectomy space was filled with the bone-core tendon ball (arrow).

Table 1. Objective Findings

\begin{tabular}{lccc}
\multicolumn{1}{c}{ Variable } & Preoperative & Postoperative & $p$-value \\
\hline Grip strength $(\mathrm{kg})$ & $13.5 \pm 6.5(72.6 \pm 32.1)(\mathrm{n}=12)$ & $15.8 \pm 5.2(100.7 \pm 18.6)(\mathrm{n}=14)$ & 0.116 \\
\hline Tip pinch $(\mathrm{kg})$ & $1.1 \pm 0.9(44.2 \pm 37.8)(\mathrm{n}=10)$ & $2.4 \pm 0.8(116.2 \pm 25.0)(\mathrm{n}=14)$ & 0.001 \\
\hline Key pinch $(\mathrm{kg})$ & $2.7 \pm 1.4(63.4 \pm 30.2)(\mathrm{n}=10)$ & $2.8 \pm 1.2(94.3 \pm 26.6)(\mathrm{n}=14)$ & 0.789 \\
\hline Radial abduction $\left(^{\circ}\right)$ & $29.2 \pm 8.4(\mathrm{NA})(\mathrm{n}=13)$ & $31.6 \pm 7.9(109.9 \pm 41.3)(\mathrm{n}=14)$ & 0.498 \\
\hline Palmar abduction $\left(^{\circ}\right)$ & $35.5 \pm 8.7(\mathrm{NA})(\mathrm{n}=13)$ & $39.3 \pm 8.6(98.9 \pm 17.6)(\mathrm{n}=14)$ & 0.157 \\
\hline Kapandji test & $\mathrm{NA}$ & $9.6 \pm 0.5(\mathrm{n}=14)$ & - \\
\hline
\end{tabular}

Values are presented as mean \pm standard deviation. The values in the parentheses are the percentage of the affected side to the contralateral side. NA: not available.

was closed tightly, and then a Kirschner wire was used for temporary fixation between the first and second metacarpal bones for 4 weeks postoperatively. A short-arm thumb spica splint was applied for 5 weeks. The patients were encouraged to move the fingers shortly after the surgery. Active motion of the thumb was permitted after the Kirschner wire removal. Formal physiotherapy was not required postoperatively.

\section{Subjective Findings}

Basal thumb pain was evaluated using a visual analog scale (VAS; 0 [no pain] to 10 [severe pain]) before surgery and at 3 years postoperatively. The Japanese version of the Disability of the Arm, Shoulder and Hand (DASH-JSSH) questionnaire was completed before surgery and at the final follow-up examination. ${ }^{12}$ Patient satisfaction with the surgery was assessed using the VAS at the final follow-up (0 [disappointed] to 10 [satisfied]).

\section{Objective Findings}

Grip strength was measured with a Smedley dynamometer (hand dynamometer; MIS, Tokyo, Japan). Tip pinch strength and key pinch strength were measured with a hydraulic pinch gauge (baseline hydraulic pinch gauges; Fabrication Enterprises, White Plains, NY, USA). The degree of volar and radial abduction was measured with a goniometer as the angle between the first and second metacarpal bones before surgery and at the final examination. Opposition was evaluated using the Kapandji test. Standard posteroanterior radiographs of the wrist were obtained for all patients before surgery, at the intermediate follow-up (a mean of 32 months [range, 19 to 56 months]), and at the final follow-up. The intermediate data were collected at the time point closest to the middle between the preoperative and final follow-up assessments. Trapezial space ratio was calculated by dividing the height of the trapezial space by the length of the first metacarpal to evaluate the first metacarpal subsidence. ${ }^{13)}$

\section{Statistical Analysis}

All data are presented as mean \pm standard deviation. Statistical significance was determined using the paired $t$-test, and $p<0.05$ was considered significant. 
Kawamura et al. Ligament Reconstruction and Tendon Interposition Using the Palmaris Longus Tendon

Clinics in Orthopedic Surgery • Vol. 11, No. 4, $2019 \bullet$ www.ecios.org

\section{RESULTS}

\section{Subjective Findings}

Compared with the preoperative level, the pain level at 3-year postoperative examination improved significantly in all the patients. The mean VAS grade improved from 8.5 (range, 5 to 10 ) to 0.4 (range, 0 to 2). The mean DASH score also improved from 44.3 (range, 23 to 71 ) to 22.6 (range, 2 to 53) in all but two patients. In one patient, the DASH score changed from 29 preoperatively to 53 at the final follow-up, because of pain in the contralateral shoulder due to adhesive capsulitis and thumb basal pain at the unoperated side. In the other patient, the preoperative DASH score was not obtained. The mean VAS score for the patients' satisfaction with the surgery was 8.7 (range, 6 to 10). The patient who had a 6 for satisfaction felt some instability in the operated thumb. Other subjective findings of this patient were improved postoperatively as the DASH score improved from 40 to 10 and pain level improved from 10 to 0 .

\section{Objective Findings}

The objective findings are shown in Table 1. There was a tendency towards an increased mean grip strength. Tip pinch strength improved significantly and there were no obvious changes in the mean key pinch strength and volar and radial abduction after surgery. The radiographic findings showed that the trapezial space ratio decreased significantly at the intermediate follow-up (Fig. 3). The ratio at the intermediate follow-up was maintained at the final follow-up (Table 2).

\section{Complications}

There was no infection or discomfort attributable to harvesting of the PL tendons or related to the FCR tendons. There were two cases of slight radial nerve irritations, which did not cause distress in the daily use of the hands.

\section{DISCUSSION}

Two recent systematic reviews showed that LRTI led to more complications and offered no additional benefits over trapeziectomy alone. ${ }^{14,15)}$ Nevertheless, LRTI is still the most common choice for many hand surgeons. ${ }^{1,2)}$ One reason for this is that some hand surgeons still believe that ligament reconstruction is essential for obtaining longterm stability at the base of the thumb after trapeziectomy. Many modifications to the LRTI procedure have been reported, ${ }^{8,16)}$ but most of them demonstrated short-term clinical results. The present study showed that the modified LRTI procedure using the PL tendon instead of the FCR tendon provided good pain relief, motion, strength, and stability without any severe complications after a mean follow-up period of 5 years.

In a previous study, favorable long-term clinical results were obtained with the conventional LRTI using the FCR tendon. ${ }^{4)}$ However, harvesting a half-FCR tendon is technically demanding and occasionally results in complications such as tendon rupture, pulling discomfort in the line of FCR tendon, and tendon adhesions. ${ }^{4,-10)}$ The PL tendon is one of the best candidates for graft material for various reconstructions of tendons and ligaments such as

\section{Table 2. Radiological Findings}

\begin{tabular}{cccc} 
Variable & $\begin{array}{c}\text { Preoperative } \\
(\mathrm{n}=14)\end{array}$ & $\begin{array}{c}\text { Intermediate } \\
(\mathrm{n}=14)\end{array}$ & $\begin{array}{c}\text { Final } \\
(\mathrm{n}=14)\end{array}$ \\
\hline Trapezial space ratio & $30.6 \pm 3.6$ & $19.3 \pm 3.1^{*}$ & $18.5 \pm 3.6^{\dagger, \ddagger}$ \\
\hline
\end{tabular}

Values are presented as mean \pm standard deviation.

${ }^{*} p<0.01$, vs. preoperative. ${ }^{\dagger} p<0.01$, vs. preoperative. ${ }^{\ddagger} p=0.20$, vs intermediate.
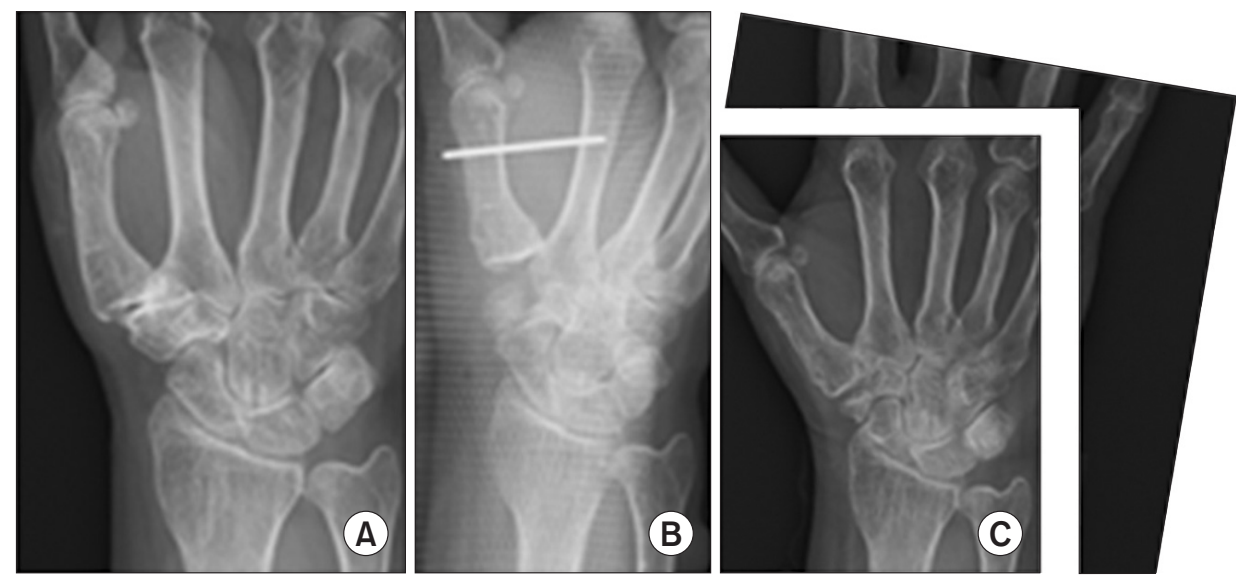

Fig. 3. Preoperative (A), 1-day postoperative (B), and 59-month postoperative (C) radiographs of a 63-year-old patient with trapeziometacarpal arthritis of Eaton and Glickel grade III. 
the flexor digitorum tendon ${ }^{17)}$ and elbow ulnar collateral ligament. ${ }^{18)}$ Harvesting the PL tendon with a tendon stripper is not a difficult procedure as long as it is performed carefully in order not to confuse the PL tendon with the median nerve. The PL tendon can be expected to work as well as a half-FCR tendon as a reconstructed ligament by securely suturing it with an interlacing suture to the FCR tendon just proximal to its insertion. Miura et al. ${ }^{16)}$ reported early functional improvement after LRTI using the PL tendon. They resected only the distal portion of the trapezium, so their procedure is not applicable for cases of Eaton and Glickel grade IV. ${ }^{11}$ Some surgeons used the entire FCR tendon to avoid complications related to using the half-FCR tendon. ${ }^{19)}$ However, passing the entire FCR tendon through the bone tunnels in the first metacarpal bones is difficult. ${ }^{20)}$ Moreover, making the drill hole in the first metacarpal bones large enough to pass the entire FCR tendon could lead to a fracture or cutout of the first metacarpals, especially in patients with small bone sizes. Our modified LRTI could be used as a salvage modality in cases of failed FCR tendon harvest if the insertion of the FCR tendon were long enough to be sutured with the PL tendon.

Our modified LRTI does not use the natural tendon bone insertion; thus, some distance exists between the insertion of the FCR tendon and the suture site of the two tendons. This could lead to concerns about the reconstructed ligament becoming loose, and instability at the base of the thumb could be induced. In the current study, we evaluated the proximal migration of the first metacarpal radiographically. The trapezial space ratio significantly decreased to $60 \%$ of the preoperative value at the intermediate follow-up. Further proximal migration was not observed at the final follow-up. These results were comparable to those of the reports of Kriegs-Au et al. ${ }^{21)}$ (35.7\%), Downing and Davis ${ }^{22)}(51.3 \%)$, Lins et al. ${ }^{6)}(69.7 \%)$, and Yang and Weiland ${ }^{13)}(79.1 \%)$. LRTI with the PL tendon could achieve acceptable stability at the base of the thumb after trapeziectomy.

The PL tendons are usually thin; therefore, the size of the tendon ball or anchovy made with the PL tendon may be too small to fill the trapeziectomy space. To overcome this problem, we used a tendon ball with a bone core made of the resected trapezium. In some cases, radiographs at the final follow-up demonstrated absorption of the bone core in the current study. However, the trapezial space ratio did not differ regardless of the absorption of the bone cores. It is reported that tendon interposition does not affect the outcome after ligament reconstruction for the treatment of osteoarthritis of the trapeziometacarpal (TMC) joint. ${ }^{5,21)}$ The current findings may provide supportive data for such reports. It is known that the incidence of unilateral PL tendon absence is $3 \%-15 \%$. $^{23,24)}$ In the current series, one patient required the use of the contralateral PL tendon because the PL tendon was absent in the operated hand. Thus, our procedure requires preoperative assessment. A patient lacking both PL tendons cannot undergo our procedure but could undergo conventional LRTI using the FCR tendon.

In a 9-year follow-up study of conventional LRTI, Tomaino et al. ${ }^{4)}$ reported that 20 of 22 patients (91\%) had complete pain relief. In the current study, complete pain relief was observed in 12 of 14 patients (86\%). Werthel and Duber ${ }^{19)}$ reported that the quick DASH score improved from 49.4 preoperatively to 22.1 postoperatively in patients treated with the LRTI using the entire FCR tendon at a mean follow-up of 3 years. Spekreijse et al. ${ }^{25}$ reported that the DASH score improved from 45.3 to 7.3 with the Weilby procedure (another LRTI using the abductor pollicis longus tendon) at a mean follow-up of 5.3 years. The postoperative DASH score in this study significantly improved from 40 to 10 at the 5 -year follow-up $(p<0.001)$. The DASH score potentially reflects the disabilities at other sites in the upper extremities; therefore, it would be better to apply other subjective measurements more specific to hand disability in future studies.

The main limitation of the current study is that it was an observational study of a single procedure. Therefore, we could not evaluate the efficacy of our method compared with other procedures for TMC osteoarthritis, such as trapeziectomy alone or trapeziectomy with tendon interposition or ligament reconstruction. However, to our knowledge, there are no other reports on the longterm clinical results of LRTI with the PL tendon. The current study showed the usefulness of our procedure for the treatment of trapeziometacarpal osteoarthritis or as a salvage procedure when the FCR tendon ruptures during the conventional LRTI procedure.

\section{CONFLICT OF INTEREST}

No potential conflict of interest relevant to this article was reported. 
Kawamura et al. Ligament Reconstruction and Tendon Interposition Using the Palmaris Longus Tendon

Clinics in Orthopedic Surgery • Vol. 11, No. 4, 2019• www.ecios.org

\section{REFERENCES}

1. Yuan F, Aliu O, Chung KC, Mahmoudi E. Evidence-based practice in the surgical treatment of thumb carpometacarpal joint arthritis. J Hand Surg Am. 2017;42(2):104-12.

2. Wolf JM, Delaronde S. Current trends in nonoperative and operative treatment of trapeziometacarpal osteoarthritis: a survey of US hand surgeons. J Hand Surg Am. 2012;37(1):77-82.

3. Burton RI, Pellegrini VD Jr. Surgical management of basal joint arthritis of the thumb. Part II: ligament reconstruction with tendon interposition arthroplasty. J Hand Surg Am. 1986;11(3):324-32.

4. Tomaino MM, Pellegrini VD Jr, Burton RI. Arthroplasty of the basal joint of the thumb: long-term follow-up after ligament reconstruction with tendon interposition. J Bone Joint Surg Am. 1995;77(3):346-55.

5. Gangopadhyay S, McKenna H, Burke FD, Davis TR. Fiveto 18-year follow-up for treatment of trapeziometacarpal osteoarthritis: a prospective comparison of excision, tendon interposition, and ligament reconstruction and tendon interposition. J Hand Surg Am. 2012;37(3):411-7.

6. Lins RE, Gelberman RH, McKeown L, Katz JN, Kadiyala RK. Basal joint arthritis: trapeziectomy with ligament reconstruction and tendon interposition arthroplasty. J Hand Surg Am. 1996;21(2):202-9.

7. Rayan GM, Young BT. Ligament reconstruction arthroplasty for trapeziometacarpal arthrosis. J Hand Surg Am. 1997;22(6):1067-76.

8. Belcher HJ, Nicholl JE. A comparison of trapeziectomy with and without ligament reconstruction and tendon interposition. J Hand Surg Br. 2000;25(4):350-6.

9. Davis TR, Brady O, Barton NJ, Lunn PG, Burke FD. Trapeziectomy alone, with tendon interposition or with ligament reconstruction? J Hand Surg Br. 1997;22(6):689-94.

10. Field J, Buchanan D. To suspend or not to suspend: a randomised single blind trial of simple trapeziectomy versus trapeziectomy and flexor carpi radialis suspension. J Hand Surg Eur Vol. 2007;32(4):462-6.

11. Eaton RG, Glickel SZ. Trapeziometacarpal osteoarthritis: staging as a rationale for treatment. Hand Clin. 1987;3(4):455-71.

12. Imaeda $\mathrm{T}$, Toh S, Nakao Y, et al. Validation of the Japanese Society for Surgery of the Hand version of the Disability of the Arm, Shoulder, and Hand questionnaire. J Orthop Sci. 2005;10(4):353-9.

13. Yang SS, Weiland AJ. First metacarpal subsidence during pinch after ligament reconstruction and tendon interposi- tion basal joint arthroplasty of the thumb. J Hand Surg Am. 1998;23(5):879-83.

14. Vermeulen GM, Slijper H, Feitz R, Hovius SE, Moojen TM, Selles RW. Surgical management of primary thumb carpometacarpal osteoarthritis: a systematic review. J Hand Surg Am. 2011;36(1):157-69.

15. Wajon A, Vinycomb T, Carr E, Edmunds I, Ada L. Surgery for thumb (trapeziometacarpal joint) osteoarthritis. Cochrane Database Syst Rev. 2015;(2):CD004631.

16. Miura T, Osuka K, Itoh S, Nakagawa T, Kawano H, Nakamura K. Early functional improvement after a modified ligament reconstruction tendon interposition arthroplasty for thumb basal joint arthritis. Hand Surg. 2008;13(3):153-8.

17. Pulvertaft RG. Tendon grafts for flexor tendon injuries in the fingers and thumb: a study of technique and results. J Bone Joint Surg Br. 1956;38(1):175-94.

18. Conway JE, Jobe FW, Glousman RE, Pink M. Medial instability of the elbow in throwing athletes: treatment by repair or reconstruction of the ulnar collateral ligament. J Bone Joint Surg Am. 1992;74(1):67-83.

19. Werthel JD, Dubert T. Use of the entire flexor carpi radialis tendon for basal thumb ligament reconstruction interposition arthroplasty. Hand Surg Rehabil. 2016;35(2):107-13.

20. Basar H, Basar B, Erol B, Tetik C. Ligament reconstruction and tendon interposition arthroplasty of the trapeziometacarpal joint with the use of the full thickness of the flexor carpi radialis tendon. Chir Main. 2012;31(6):331-6.

21. Kriegs-Au G, Petje G, Fojtl E, Ganger R, Zachs I. Ligament reconstruction with or without tendon interposition to treat primary thumb carpometacarpal osteoarthritis: a prospective randomized study. J Bone Joint Surg Am. 2004;86(2):209-18.

22. Downing ND, Davis TR. Trapezial space height after trapeziectomy: mechanism of formation and benefits. J Hand Surg Am. 2001;26(5):862-8.

23. Sebastin SJ, Puhaindran ME, Lim AY, Lim IJ, Bee WH. The prevalence of absence of the palmaris longus: a study in a Chinese population and a review of the literature. J Hand Surg Br. 2005;30(5):525-7.

24. Thompson NW, Mockford BJ, Cran GW. Absence of the palmaris longus muscle: a population study. Ulster Med J. 2001;70(1):22-4.

25. Spekreijse KR, Selles RW, Kedilioglu MA, et al. Trapeziometacarpal arthrodesis or trapeziectomy with ligament reconstruction in primary trapeziometacarpal osteoarthritis: a 5-year follow-up. J Hand Surg Am. 2016;41(9):910-6. 